\title{
》TYPISCH DEUTSCHE« POPULÄRE MUSIK HEUTE. EINE ANNÄHERUNG
}

\author{
Thomas Phleps
}

\author{
»Ihr wünscht euch alle so ein'n Typen wie mich. \\ Kein Problem, ich bleib' einfach typisch ich « \\ (Bushido: »Typisch Ich«, 2004). \\ »Was der deutsche Geist sein könnte, wer hätte nicht schon \\ darüber seine schwermütigen Gedanken gehabt!«
}

(Nietzsche 1967a: 358).

Was ist »typisch deutsch«? Neuere Studien bilanzieren, dass es den »typisch Deutschen« nicht gibt resp. es neben der (falschen) Fassade des »typisch Deutschen « ein diese Stereotypen konterkarierendes, wahres Leben im untypisch deutschen Privaten gebe ${ }^{1}$ - zur Klärung von »typisch deutsch«, diesem Beinahe-Kompositum, tragen diese Studien leider wenig bei. Lassen Sie mich daher eingangs die Problematik zumindest anreißen:

Das Typische ist nichts einer Sache, einem Sachverhalt, einer Handlung, einem Verhalten oder Denken Inhärentes, sondern ohne Zweifel eine Zuschreibung - welcher Art freilich, ist einigermaßen unklar. Zu denken wäre an Max Webers Idealtypus², Hermann Bausingers Stereotypen (mit der Stei-

1 Cf. die große Markt-Media-Studie »VerbraucherAnalyse 2010 « der Axel Springer AG und der Bauer Media Group (http://www.verbraucheranalyse.de/downloads /51/VA_2010_Vortrag_typisch_deutsch.pdf; letzter Zugriff 5.4.2014) oder die Studie »Das Doppelleben der Deutschen« der OeTTINGER Brauerei von 2012 (http://www.rheingold-salon.de/grafik/veroeffentlichungen/Deutschland_Studi e_reader_2012-09-19.pdf; letzter Zugriff 5.4.2014).

2 Der Idealtypus »wird gewonnen durch einseitige Steigerung eines oder einiger Gesichtspunkte und durch Zusammenschluss einer Fülle von diffus und diskret, hier mehr, dort weniger, stellenweise gar nicht, vorhandenen Einzelerscheinungen, die sich jenen einseitig herausgehobenen Gesichtspunkten fügen, zu einem in sich einheitlichen Gedankenbilde. In seiner begrifflichen Reinheit ist dieses Gedankenbild nirgend in der Wirklichkeit empirisch vorfindbar [...]. [So] erwächst die Aufgabe, in jedem einzelnen Falle festzustellen, wie nahe oder wie fern die Wirklichkeit jenem Idealbilde steht« (Weber 1991: 73f.). 
gerung Vorurteil) oder an Bertolt Brechts dezidiert marxistische Deutung »des Worts >typisch« als »geschichtlich bedeutsam« im Sinne von wichtig »für den Fortschritt der Menschheit, das heißt für den Sozialismus wichtig « (Brecht 1967: 531) ${ }^{3}$. Hier klingt übrigens deutlich die ursprüngliche Bedeutung des griechischen typikós - bildlich, vorbildlich - an.

Laut Georg Lukács (1967: 341) hat der Typus den »Charakter der Besonderheit « - als Beispiel nennt er die Marxsche Konzeption der »Charaktermaske ${ }^{4}-$; die Besonderheit wiederum wird gefasst »als Spielraum, als Kraftfeld zwischen Allgemeinem und Einzelnem, als organisierende Mitte ihrer widerspruchsvollen Bezogenheit aufeinander« (ib.: 365f.). Im Prinzip rekurriert Lukács in seiner Typus-Definition auf die Kategorie des »Charakteristischen«, die erstmals 1797 von Aloysius Hirt in Schillers Horen für die ästhetische Diskussion bereitgestellt wurde. Stante pede arbeiteten sich die 'Klassiker< an dieser »Individuellheit der Bedeutung « (Hirt 2008: 38) im Melting Pot von Wahrem und Wahrscheinlichem ab. ${ }^{5}$

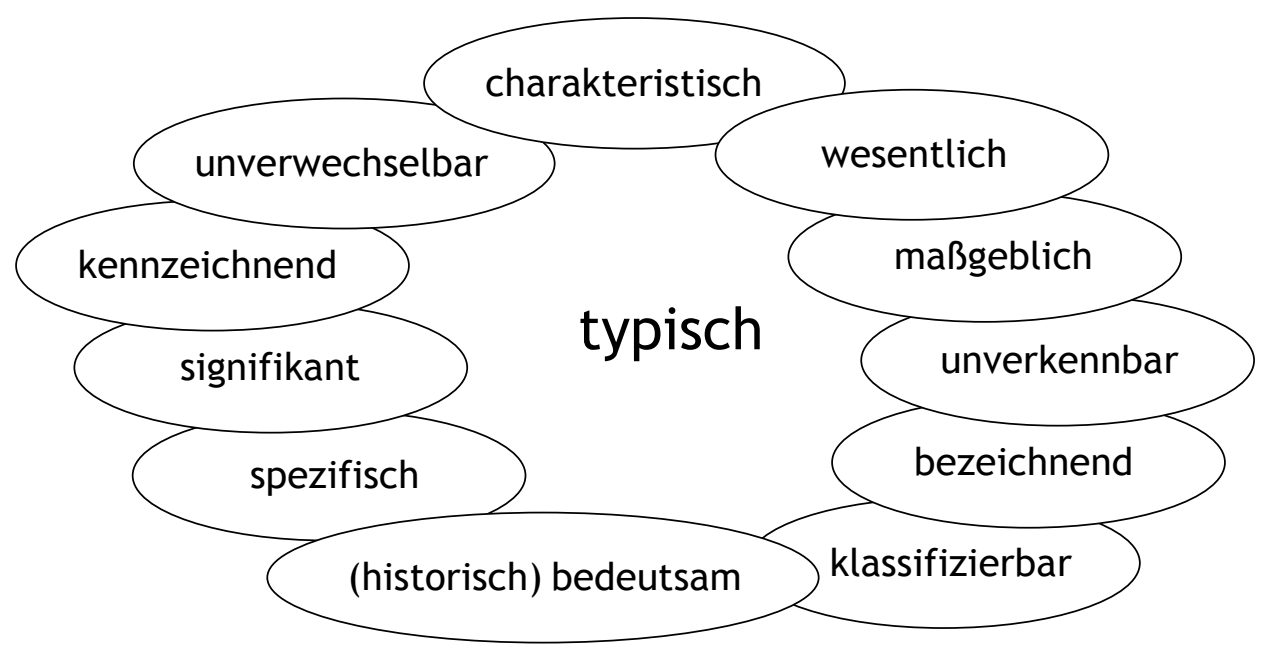

3 Und »dieser Begriff gestattet, auch scheinbar winzige, seltene, übersehene Vorkommnisse sowie unscheinbare, oft oder selten vorkommende Menschen ans Licht der Dichtung zu ziehen « (Brecht 1967: 531).

4 Als »die gedankliche Zusammenfassung jener notwendigen Eigenschaften, z.B. des Kapitalisten, die aus seiner in der Produktion eingenommenen Stellung zwangsläufig erfolgen, die darum aus den ökonomischen Gesetzlichkeiten abgeleitet sind und nicht eine bloße Summierung oder Synthese seiner psychologischen Zergliederung bilden« (Lukács 1967: 341). Marx (1973: 100) sagt, »daß die ökonomischen Charaktermasken der Personen nur Personifikationen der ökonomischen Verhältnisse sind, als deren Träger sie sich gegenübertreten «.

5 Cf. bspw. Goethes Dialog Über Wahrheit und Wahrscheinlichkeit der Kunstwerke (1798). Zu diesem Komplex cf. Costazza 2006. 
Summiert man die Erklärungsansätze, so ist »typisch« vor allem die Zuschreibung eines Bündels an Denk-, Schreib- und Verhaltensweisen, die charakteristisch, wesentlich, maßgeblich, unverkennbar, unverwechselbar, kennzeichnend, bezeichnend, signifikant, spezifisch, klassifizierbar und (historisch) bedeutsam sind - ja, für was oder wen: für deutsch, das Deutsche, den Deutschen, Deutschland? Hermann Bausinger (2000: 21) umgeht diese Problematik elegant, indem »typisch deutsch « aus seiner Sicht das ist, »was Nichtdeutsche für typisch deutsch halten« - auch wenn er einschränkt: »zunächst einmal«, frage ich mich zunächst einmal, was »Nichtdeutsche« sind. Bausinger nennt zunächst einmal Chilenen, Franzosen, Dänen, aber wie sieht es mit Österreichern, Südtirolern, deutschsprechenden Schweizern aus? Und auch, wenn es in Deutschland selbst seit über zwanzig Jahren keine BRD- und DDR-Deutschen mehr gibt, gibt es auch hierzulande weiterhin zwei Sorten Deutsche - zumindest was die Löhne und Renten angeht.

Wo also beginnt und wo endet das oder der/die Deutsche? Heißt »deutsch sein«, um den berühmt-berüchtigten Satz Richard Wagners zu zitieren, »eine Sache um ihrer selbst willen treiben ${ }^{6}{ }^{6}$ oder halten wir es mit dem Abtrünnigen Nietzsche: »wie viel Bier ist in der deutschen Intelligenz!« (Nietzsche 1967a: 358) - ein Ausruf, kein Fragesatz! Typisch deutsch im Sinne von kennzeichnend ist jedenfalls, dass - ebenfalls Nietzsche - bei den Deutschen »die Frage >was ist deutsch? niemals ausstirbt « (Nietzsche 1967b: 131).

Ausgestorben, d.h. zumindest in der Musikwissenschaft ausgestorben ist allerdings seit oder besser mit dem Abtreten der letzten NS-Infizierten die Frage, was das >Deutsche< in der Musik sei. ${ }^{7}$ Das Festhalten an der Denkfigur einer »Vorherrschaft der deutschen Musik « scheint hingegen von all dem unberührt - ein Festhalten, durch das sich laut Anselm Gerhard (2006: 27) »die internationale Musikwissenschaft in einen Zustand der intellektuellen Sklerose befördert « habe. Hier ist - versteht sich - natürlich ausschließlich von sogenannter E-Musik die Rede. In den einzelnen Sektoren populärer Musik kann von einer solchen Vorherrschaft kaum die Rede sein.

6 So kennt man diesen Satz und so steht er in Büchmanns Geflügelten Worten, nicht aber bei Wagner! Cf. Büchmann 1941: 220; Wagner 1914: $96 \mathrm{f}$.

7 Bernd Sponheuer hat die beiden Begriffsgebilde dieses (letztlich substanzlosen) Funktionsbegriffs herausgearbeitet: Das Deutsche in der Musik als das »spezifisch >Deutsche « und als »die große Synthese « bzw. »universale >Weltmusik « (Sponheuer 2001: 129f.). Und er weist darauf, dass die Diskussion dieser beiden »Begriffe des >Deutschen<, der privative und der universalistische « Mitte des 19. Jahrhunderts »in ihren wesentlichen Grundzügen « (ib.: 131) angeschlossen ist. Die NS->Wissenschaft $<$ hat dieser fundamentalen Dichotomie nichts hinzuzufügen, sondern erweist sich wie so oft allein »als wendige Rezeptions- und Reartikulationsagentur« (ib.: 148). 
Sie merken, ich komme langsam zu meinem Thema - und ich muss sogleich zugeben, dass mir die Beschäftigung mit aktueller »typisch deutscher« populärer Musik wenig Freude bereitet hat. Selbst wenn vorauszusetzen ist, dass Popularmusikforschung keine sgehobene< resp. in elaboriertem Code vermittelte Fanrezeption sein kann und darf (was freilich, betrachtet man bspw. die deutschsprachige Literatur zu HipHop und vor allem Metal, mitunter vergessen zu werden scheint), so ist die Auseinandersetzung mit Phänomenen aus den Sektoren Jazz, Blues oder Rock doch weitaus angenehmer und anregender als ein analytisches Einlassen auf das aktuell Populäre, bei dem man in weiten Teilen tatsächlich genötigt zu sein scheint, alle Hoffnung fahren zu lassen.

Oder peinlich berührt zu sein resp. sich fremdzuschämen, wie es heute heißt. Denn ist es nicht peinlich, wenn ein an die 70-Jähriger sonnenbebrillt im Angesicht zweier ausgesprochen gut aussehender, vor allem aber ausgesprochen junger Frauen den Elvis-Hüftschwung zu exerzieren versucht? ${ }^{8}$ Aber Udo Lindenberg war schon als halb so Junger peinlich, als er $1981 \mathrm{der}$ so genannten No-Future-Generation in gewohnt holpriger Pseudo-Jugendsprachlichkeit den Marsch sang: »Du bist erst 15 - auf deiner Jacke steht: NO FUN! / Wär' nichts zu ändern, fängst auch erst gar nicht mehr mit irgendwas an« (»No Future?«).

Und ist es nicht peinlich, wenn ein in die fetten Jahre gekommener Bochumer auf einer Riesenbühne sein Konsonantendeutsch als Rockkonzert für jedermann zelebriert? Aber Herbert Grönemeyer war schon 1984 peinlich, als er samt seiner ausgebufften Studiotruppe auf der Woge der Neuen Deutschen Welle (und Ina Deters »Neue Männer braucht das Land«) mitschwamm und so »blöde«, wie es nur »Männer « können, fragte, wann »ein Mann ein Mann« sei.

Überhaupt die NDW: So viele gute Sachen und übrig geblieben ist der Bodensatz. BAP bspw. mit ihrem Fantasie-Dialekt sprechenden Sprecher Wolfgang Niedecken, der sich inzwischen zum deutschen Bob Dylan gekrönt hat. Das Gebaren dieses öffentlichen Schlaganfallpatienten ist peinlich, aber beileibe nicht der Gipfel der Peinlichkeiten. In dessen Nähe gelangen wir mit der alterslos schwarz-blond umrahmten Gesichtsmaske Heinz Georg Kramm, genannt Heino, dessen gefühlte Jahrhunderte währende Anziehungskraft mir auf ewig ein Rätsel bleiben wird und der mit demselben

8 So zu sehen im 60-minütigen Zusammenschnitt dreier Konzerte in der Kölner Lanxess-Arena zum Abschluss der Lindenberg-Tournee 2013 am 30./31. März und 2. April, die $\gg$ Das Erste « im Anschluss an eine NDR-Dokumentation zu Udo Lindenberg aus der Reihe »Pop-Legenden« am 31. Juli 2013 ab 23:30 Uhr ausstrahlte. 
todbringenden Ernst einst alle drei Strophen des Deutschlandliedes nebst einigen akkuraten $\mathrm{HJ}$-Liedern einsang, wie er heute den Ärzte-Song »Junge« massakriert.

Warum erwähne ich diese altgebliebenden Jungstars? Nun, sie alle haben in jüngster Zeit Charterfolge gefeiert und auf eine Einsicht in diese Charts möchte ich mich im Folgenden beschränken - auch wenn die aktuelle populäre Musik bei weitem nicht nur in den Charts zu finden ist, sondern auch und eigentlich vor allem in den Vergnügungszentren der Jugend, der Erwachsenen und der Greise, sprich den Discos, den Konzerthallen und -stadien sowie den Radio- und Fernsehapparaten. Aber natürlich ist es mehr als vermessen, über den aktuellen Stand »typisch deutscher« populärer Musik berichten zu wollen - zu vielfältig ist das System, zu knapp die Zeit und (was häufig unter den Tisch fällt) zu wenig informiert der Verfasser.

Noch kurz vorweg: Deutsche populäre Musik ist zunächst rein geographisch verortet und meint alle populäre Musik, die im deutschsprachigen Raum produziert wurde und/oder in der Deutsch gesungen wird bzw. in einem dem deutschen Sprachraum zugehörigen Dialekt oder einer restringierten Version wie bspw. Kanak Sprak oder auch eher Rudimentärem wie Bobbele?.

Die von mir herangezogene Chartliste - es ist tatsächlich eine einzige, nämlich die von den fleißigen Wichteln des legalen Daten(an)sammlungsprojekts Wikipedia bereitgestellte Liste der Nummer Eins-Alben in Deutschland $2013^{10}$ und ich habe - dies nebenbei - diese Liste ganz zufällig zum Ausgangspunkt gewählt - aber sie macht, so scheint es, die Sache so einfach wie deutlich:

9 »Ich sag ja auch ganz klar in diesem Buch, dass ich stolz Deutscher zu bin« (Boris Becker im Interview mit dem Hessischen Rundfunk am 10. Oktober 2013 auf der Frankfurter Buchmesse; cf. http: //www.youtube.com/watch?v=eaG4gQ c_m4k, letzter Zugriff 5.4.2014).

10 Cf. http://de.wikipedia.org/wiki/Liste_der_Nummer-eins-Hits_in_Deutschland_ (2013) (letzter Zugriff 5.4.2014; die in eckiger Klammer hinzugefügten Genrezuweisungen stammen vom Autor). Media Control, laut eigener Homepage »führender Dienstleister im Bereich Medieninformationen« (http://www.mediacontrol.de, letzter Zugriff 5.4.2014), stellt seit einiger Zeit kein Archiv mehr der von diesem Kontrollmedium wöchentlich zusammengestellten, auf Tonträger- wie Download-Käufen basierenden Charts bereit. 
Nummer Eins-Alben in Deutschland 2013 von Woche 1 bis 52

1. Mrs. Greenbird: Mrs. Greenbird

2. Helene Fischer: Für einen Tag - Live 2012

[FolkPop]

3. Mrs. Greenbird: Mrs. Greenbird

[Schlager]

4.-5. Andrea Berg: Abenteuer - 20 Jahre Andrea Berg

[FolkPop]

6. Matthias Reim: Unendlich

[Schlager]

7. Heino: Mit freundlichen Grüßen

[Schlager]

8. Kollegah \& Farid Bang: Jung Brutal Gutaussehend 2

[Schlager]

9.-11. Heino: Mit freundlichen Grüßen

[HipHop]

12. David Bowie [UK]: The Next Day

[Schlager]

[Pop]

13. Justin Timberlake [US]: The 20/20 Experience

[Pop]

14.-15. Depeche Mode [UK]: Delta Machine

[Pop]

16. Volbeat [DK]: Outlaw Gentlemen $\mathcal{E}$ Shady Ladies

[Heavy Metal]

17. Prinz Pi: Kompass ohne Norden

[HipHop]

18. Frei.Wild: Feinde deiner Feinde

[RechtsRock]

19. Deep Purple [UK]: Now What?!

[Rock]

20. Reinhard Mey: Dann mach's gut

[Liedermacher]

21. Santiano: Mit den Gezeiten

[Schlager]

22. Daft Punk [FR]: Random Access Memories

[House]

23. Tim Bendzko: Am seidenen Faden

[Singer-Songwriter]

24. Xavier Naidoo: Bei meiner Seele

[Pop: "R\&B"]

25. Black Sabbath [UK]: 13

[Heavy Metal]

26. Amigos: Im Herzen jung

[Schlager]

[HipHop]

27. Genetikk: D.N.A

[Electro-Pop]

28. Frida Gold: Liebe ist meine Religion

[HipHop]

RAF 3.0: Hoch

[HipHop]

31. Powerwolf: Preachers Of The Night

["Power-Metal"]

32. Hansi Hinterseer: Heut' ist dein Tag

[Schlager]

33. Alligatoah: Triebwerke

[HipHop]

34. Helge Schneider: Sommer, Sonne, Kaktus!

[Schneider-Pop]

35. Saltatio Mortis: Das schwarze $1 \times 1$

[Mittelalter-Rock]

36. Eko Fresh: Eksodus

[HipHop]

37. Schiller: Opus

38. Andrea Berg: Atlantis

39. Die Ärzte: Die Nacht der Dämonen - Live

[Electro-Pop]

[Schlager]

[PunkRock]

40. Andrea Berg: Atlantis

[Schlager]

41. Casper: Hinterland

[HipHop]

42.-44. Helene Fischer: Farbenspiel

[Schlager]

45. Prince Kay One: Rich Kidz

[HipHop]

46.-47. Eminem [US]: The Marshall Mathers LP 2

48. Robbie Williams [UK]: Swings Both Ways

[HipHop]

[Pop]

49. Frei.Wild: Still

[RechtsRock]

50. Sido: $30-11-80$

[HipHop]

51.-52. Robbie Williams [UK]: Swings Both Ways

[Pop] 
Deutlich ist, dass bis auf Mrs. Greenbird und Powerwolf (beide wohl Genrebedingt) die deutschen Musiker in den Charts deutsch sprechen und singen mitunter, wie etwa bei Frida Gold, durchsetzt mit Sextaner-Englisch. Und es ist deutlich, dass in diesen Album-Charts (mindestens) zwei Parallelwelten koexistieren: die Welt der Älteren und die der Jugendlichen, die in den Album-Charts vor allem die deutschen Rapper auf Platz Eins pushen und die übrigens unangefochten und allein für die Single-Charts zuständig sind, sodass sich hier ein gänzlich anderes Bild zeigt. In den insgesamt 52 Wochen stehen lediglich zwei Wochen zwei >deutsche< Titel auf Platz Eins:

Nummer Eins-Singles in Deutschland $2013^{11}$

1. Rihanna [US]: »Diamonds «

(10 Wochen auf Platz 1)

2. Psy [KR]: »Gangnam Style«

(3 Wochen)

3.-11. Will.i.am \& Britney Spears [US]: »Scream \& Shout«

(9 Wochen)

12.-16. Passenger [UK]: »Let Her Go «

(5 Wochen)

17.-18. Pink feat. Nate Ruess [US]: »Just Give Me A Reason«

(2 Wochen)

19.-20. Capital Cities [US]: »Safe And Sound «

(2 Wochen)

21. Beatrice Egli [CH]: »Mein Herz«

(1 Woche)

22.-23. Daft Punk feat. Pharrell Williams [FR/US]: „Get Lucky«

(2 Wochen)

24.-27. Robin Thicke feat. T.I. \& Pharrell [US]: »Blurred Lines«

(4 Wochen)

28. Cro [DE]: »Whatever «

(1 Woche)

29.-38. Avicii [SE]: »Wake Me Up«

(10 Wochen)

39.-41. Jason Derulo feat. 2 Chainz [US]: »Talk Dirty«

(3 Wochen)

42. James Blunt [UK]: »Bonfire Heart«

(1 Woche)

43. Klingande [SE]: »Jubel«

(1 Woche)

44. Avicii [SE]: »Hey Brother«

(1 Woche)

45.-50. Klingande [SE]: »Jubel«

(6 Wochen)

51. Faul \& Wad Ad vs. Pnau [F]: »Changes«

(1 Woche)

52. Pitbull feat. Kesha [US]: »Timber«

(1 Woche)

Für die Album-Charts aber zielt es völlig ins Leere bzw. ist es reinste Demagogie, wenn Philipp Burger, Sänger der Band Frei.Wild, »die Deutschen« auffordert, »endlich [zu] begreifen, dass nicht nur englische Musik gefallen kann! Es [gebe] kein Land dieser Erde, was derart auf fremdsprachige Musik steht wie eben Deutschland! « (Kuboth 2005). Das also ist typisch deutsch auf Fremdsprachiges stehen. Burger muss es wissen, er guckt schließlich ich erinnere an Bausingers Klärungsansatz - als Südtiroler von außen drauf. Aber zu Frei.Wild, die der anscheinend nicht nur - Achtung: Doppeldeutigkeit - : verblendete Kollege Heino nicht zu kennen meint (cf. Summen 2013)

11 Cf. http://de.wikipedia.org/wiki/Liste_der_Nummer-eins-Hits_in_Deutschland_ (2013) (letzter Zugriff 5.4.2014). Die zehn resp. drei Wochen auf Platz Eins der ersten beiden Singles ergeben sich aus dem >Überhang v von 2012. 
und die in diesem Jahr auch zweimal die Album-Charts anführten - und hier scheint das Wort »führen « an der rechten Stelle (zumal sie die Charts beim ersten Mal - kaum zu glauben, aber wahr: in der 18 . Woche dieses Jahres anführten), aber zu Frei.Wild ist ja schon andernorts einiges gesagt worden ${ }^{12}$, sodass ich mir weitere Kommentare sparen kann.

Burger liegt übrigens auch falsch, wenn man sich nicht nur die ersten, sondern die ersten zehn Plätze der Album-Charts 2013 anschaut. ${ }^{13}$ Und er liegt ebenso in der Rückschau falsch: 2011 waren 17 deutsche Interpreten resp. Alben in 30 Wochen auf Platz Eins, hingegen 16 ausländische in 22 Wochen; 2012 waren es 18 deutsche Interpreten resp. Alben in 35 Wochen und 12 ausländische in 17 Wochen. ${ }^{14}$ Und 2013 steht in den 52 Wochen, die hier aufgelistet sind, nur ganze 13 Wochen kein Album deutscher resp. deutschsprachiger Musiker auf Platz Eins:

- In den 39 >deutschen< Wochen mit aktueller deutscher Musik sind 31 verschiedene Alben von 27 verschiedenen Interpreten auf Platz Eins.

- Von diesen 31 verschiedenen Alben stammen 9 aus dem Schlager- und 10 aus dem HipHop-Bereich, die restlichen 12 sind eher poppig (6), rockig (5) oder -: Helge Schneider, aber dazu später mehr...

- Die 9 Schlageralben halten sich häufig länger in den Charts: insgesamt 16 Wochen, die 10 HipHop-Alben jeweils nur eine Woche.

Bemerkenswert und vielleicht sogar typisch für die aktuelle deutschsprachige Rapmusik ist eine große Vielfalt der Interpretationen, bei denen das thematische Spektrum von einer Hymne des Grasrauchens ${ }^{15}$ über mathematischen Aporien ${ }^{16}$ bis zur Problematisierung der Konsequenzen des Erwachsenseins ${ }^{17}$ reicht. Und natürlich bleibt es unvermeidlich, »Albumlänge[n] lang Ohrenzeuge des ausdauernden Schwengel-Geschwenkes sein zu müssen«, wie kürzlich auf laut.de anlässlich der neusten »thematischen Einöde« Lil Waynes zu lesen stand (Fromm 2013).

Während die Spielarten dieser nachpubertären Testosteronitis bei allen handwerklichen Unzulänglichkeiten selbst beim »Zuhälterrap « (Selbstbeschreibung!) des Jurastudenten Kollegah (alias Felix Blume) und seines Kollegen Farid Bang eine gewisse Freude an der Sache vermitteln, exerziert

12 Cf. kursorisch http://de.wikipedia.org/wiki/Frei.Wild (letzter Zugriff 5.4.2014); auch Thorsten Hindrichs' Beitrag in diesem Band.

13 Cf. http://www.musicline.de/de/charts/longplay (letzter Zugriff 5.4.2014).

14 Cf. http://de.wikipedia.org/wiki/Liste_der_Nummer-eins-Hits_in_Deutschland_ (2011) und http://de.wikipedia.org/wiki/Liste_der_Nummer-eins-Hits_in_Deuts chland_(2012) (letzter Zugriff 5.4.2014).

15 Cf. Titelsong von Genetikk: D.N.A.

16 »Leb dein Leben zum Quadrat« heißt es im Titelsong von RAF 3.0: Hoch 2.

17 Cf. Titelsong von Prinz Pi: Kompass ohne Norden. 
der NWA-Propagandist Shindy die Depravation von Anspruch zu Beschäftigungslosigkeit - N.W.A. = Niggaz Wit Attitudes Nie wieder arbeiten ${ }^{18}-$ und die Einübung in Hass und Hetzerei. Assistiert von Bushido macht er "Stress ohne Grund «, und es ist vor allem die verschärfte Bad Boy-Attitüde des Integrations-Preisträgers, ${ }^{19}$ die das Album auf die Liste jugendgefährdender Medien brachte - und zwar, auch wenn das schwer zu verstehen ist, eine Woche, bevor es in den Charts auf Platz Eins landete. In »Stress ohne Grund erteilt der bekennende AfD-Wähler (cf. fro 2013) Bushido u.a. die Lizenz zum Töten des FDP-Politikers Serkan Tören, der Grünen Claudia Roth und seines einstigen Kumpels Prince Kay One (der trotz - oder gar wegen? dieser Anwürfe 15 Wochen später mit seinem dritten Solo-Album Rich Kidz auf Platz Eins der Album-Charts gelangte):

Shindy feat. Bushido: »Stress ohne Grund «, 2. Strophe (Bushido)

Halt die Fresse, fick die Presse, Kay, du Bastard bist jetzt vogelfrei.

Du wirst in Berlin in deinen Arsch gefickt wie Wowereit.

Yeah, fick die Polizei, LKA, BKA.

Meine Jungs verticken Elektronik so wie Media Markt.

Jeden Tag im Fadenkreuz, ich zeig dir, wie der Hase läuft.

Arabisch-deutsche Sippe und ich jage euch, ihr Partyboys.

Du versteckst dich, doch ich finde dich wie Google Maps.

Ich verkloppe blonde Opfer so wie Oli Pocher,

Ich mach Schlagzeil'n, fick deine Partei, yeah,

Und ich will, dass Serkan Tören jetzt ins Gras beißt, yeah yeah

[zugleich Schussgeräusche],

Was für Vollmacht, du Schwuchtel wirst gefoltert.

Ich schieß auf Claudia Roth und sie kriegt Löcher wie ein Golfplatz. ${ }^{20}$

Im Video ${ }^{21}$ läuft dazu als eine Art paralleler Gewaltexzess das Kidnapping eines Mannes im Kofferraum eines HipHop-typischen Protzautos und zum Schluss, nachdem Bushido noch schnell mal ein Handy-Foto des Opfers gemacht hat, die Inbrandsetzung des benzinüberschütteten Autos durch einen

18 N.W.A. = »Niggaz Wit Attitudes $«$ : die äußerst erfolgreiche HipHop-Crew um Dr. Dre und Ice Cube Ende der 1980er, Anfang der 1990er Jahre.

19 Bushido erhielt 2011 vom Burda-Verlag den Bambi für Integration mit der Begründung, er setze »sich ein gegen Gewalt und für ein respektvolles Miteinander in einer multikulturellen Gesellschaft « (Korn 2011). Der Aufschrei in der (von Burda unabhängigen) Presse war groß; interessanterweise hat nach der Verleihung (und als einziger Preisträger) Heino sein 1990 erhaltenes Rehkitz zurückgegeben.

20 Leicht korrigiert zit. n. BPjM 2013: 10.

21 Das Video ist übrigens weiterhin »unzensiert « und unter mehreren Adressen im Netz zugänglich; cf. bspw. http://www.youtube.com/watch?v=uPSf-u-_md0 (letzter Zugriff 5.4.2014). 
muskelbepackten Adlatus der beiden Rapper. Hinzu kommt noch ein Schuss Homophobie und fertig ist der Battle-Rap, in dem der »Musikkünstler Bushido « - so sein Verfahrensbevollmächtigter - nur »die Mittel genutzt [habe], die ihm als Rapper zur Verfügung stünden« - Worte nämlich, die man aber »keinesfalls [...] wörtlich zu nehmen« habe (BPjM 2013: 4). Sprachliche Gewalt, die man nicht wörtlich nehmen darf... Es ist schon erschreckend, wie man hier zumal angesichts des »mafiös-violenten Kontext[es]« (ib.: 10) ${ }^{22}$ in kulturkonservativen Stress zu geraten droht - gewiss nicht grundlos, aber die Sprachbarrieren zu den Vorbildern der deutschen Gangsta-Rapper haben uns da doch wirksamer vor den eigenen Zweifeln am Grundrecht künstlerischer Freiheit bewahrt. ${ }^{23}$

Und natürlich ist Bushido vor allem ein großer sSkandalisierungskünstler - ganz im Gegensatz zu den Vertretern des Pop- und Schlagersektors: Hier hatten die deutschen Charts (West wie Ost!) früher vor allem adrette Schwiegersöhne im Programm, heute sind es forever jung gebliebene Stiefväter bzw. im Amigos-Fall: Großonkels - >liebe Onkels darf man, nicht dass der eine oder die andere auf falsche Gedanken kommt, nicht sagen, schließlich haben die beiden Altgebliebenen mehrere Lieder gegen Kindesmissbrauch fabriziert und engagieren sich im Weißen Ring.

Dass die Album-Charts (sieht man von den Rappern ab) der grassierenden Vergreisung der Bevölkerung in Deutschland Rechnung tragen - selbst bei Frida Gold sehen neben der sehens-, aber leider nicht hörenswerten Sängerin Alina Süggeler die Musiker ziemlich alt aus -, dass auch die Charts zu vergreisen drohen, muss ja eigentlich nicht heißen, dass der Musik jeglicher Esprit und Elan abhanden kommt. Heißt es aber leider! Die Musik all dieser Pop- und Schlagermusik ist langweiliger und vorhersehbarer als eine Packung Knäckebrot. Die persönliche Note, wenn ich das Wenige an Unterscheidbarem mal so nennen darf, diese Note findet sich nicht in den großen formalen Strukturen: durchgängig wird ein sauberes Erscheinungsbild gepflegt, durchgängig wird unsauber gereimt - selbst bei dem, dessen Name eine gewisse Verpflichtung in sich birgt ${ }^{24}$ :

22 Kay-One steht wg. seiner Äußerungen über die Geschäftsbeziehungen von Bushido zu einem libanesisch-palästinensischen Berliner Clan seit Wochen unter Polizeischutz (cf. stern exklusiv 2013).

$23 \mathrm{Cf}$. zur »Kunstfreiheit « die vom Bundesverfassungsgericht dekretierten und in der BPjM-Entscheidung angeführten Auffassungen, dass »das Wesentliche der künstlerischen Betätigung [...] die freie schöpferische Gestaltung « sei und jetzt kommt's: »das Wesentliche eines Kunstwerks [...], dass bei formaler, typologischer Betrachtung die Gattungsanforderungen eines bestimmten Werktyps erfüllt sind (BPjM 2013:14f.).

24 Am schönsten reimt Reim in der Parodie seines Erfolgstitels »Verdammt ich lieb Dich« für einen Autoverleiher-Werbespot: »Verdammt, ich hab' nichts, ich miet' 
Matthias Reim: »Wir sind unendlich«, Refrain

Wir sind unendlich,

Wir sind unvergänglich.

Und denk bitte nicht,

Dass ich das nur so sag'

Und morgen früh wieder vergessen hab'.

Wir sind unendlich,

Wir sind unzertrennlich.

Und das rede ich nicht bloß so daher.

Nein, ich weiß, dass es stimmt,

Dass wir unendlich sind.

Und durchgängig wird ein kaum variiertes Strophe-Refrain-Schema exekutiert. Die persönliche Note ist vorrangig im Image abgelegt, das wiederum an Unglaubhaftigkeit und Unglaublichkeit kaum zu überbieten ist - für den Außenstehenden, versteht sich. Es gibt den verwegenen Matthias Reim oder den bodenständigen Hansi Hinterseer, es gibt den Meister der »herben Molltöne ${ }^{25}$ Heino (der alle anderen >gesanglich< um Längen schlägt), und es gibt $H F$ und $A B$, die mit den Initialen höchster lyrischer Provenienz ${ }^{26}$ ausgestatteten beiden weiblichen Megastars, die facettenreich-königliche Helene Fischer (die Ende 2013 mit 3 Singles und 3 Alben in den Charts vertreten ist, eines davon seit über 3 Jahren) ${ }^{27}$ - und die schärfste Frau des deutschen Schlagers, Andrea Berg: Ihre Compilation Abenteuer - 20 Jahre Andrea Berg stand Anfang des Jahres auf dem Gipfel - und wer sich durch diese zwei CDs durchhört, könnte meinen, er sei in einen Fortsetzungsroman von Gottfried August Bürger geraten: Frau Berg ist umgeben von Kerlen, die lügen bis zu tausend Mal pro Person, wie im ersten dieser Lügen- resp. BelügenSongs, dessen Einstieg eine meiner ewig währenden Lieblingszeilen bringt (Sie dürfen raten, welche es ist): ${ }^{28}$

bei Sixt!«. Hintergrund ist seine Millionenpleite, die er allerdings 2010 nach nur vier Jahren durch ein verkürztes Insolvenzverfahren kompensieren konnte (cf. Jüttner 2007; Gothe-Syren 2010).

25 Noch schöner im Zusammenhang: »Die leichte Wehmut und die herben Molltöne von Heinos Liedern sind ein gesunder Gegenpol zur modischen Beathysterie (Covertext der Heino-LP Wir wollen zu Land ausfahren. Fono-Ring SFGLP 78 009; 1967).

26 Reiner Zufall natürlich, dass exakt dieselben Initialen die bedeutungstragenden Töne in Bergs Lyrischer Suite bilden, hier allerdings die des Komponisten Alban Berg und seiner kurzzeitigen Geliebten Hanna Fuchs-Robettin.

27 Und die anscheinend derart mit Preisen überhäuft wird, dass sie sie zu verwechseln beginnt - am Ende der 65. Bambi-Verleihung verkündete sie: »Das war der Echo 2013 « (cf. Weimer/Vogel 2013).

28 »Du hast mich tausendmal belogen« belegt übrigens auch Platz Eins der GEMA 
Andrea Berg: »Du hast mich tausendmal belogen«, 1. Strophe

Du brauchst das Gefühl, frei zu sein.

Niemand, sagst du, fängt dich ein.

Doch es war total Liebe pur.

Manchmal frag' ich mich: Warum nur du?

Warum nur Dur? Ja, in Moll stehende Schlager haben Seltenheitswert, und zumal »Abenteuer«, wie sie die best-ausgezogene Frau der deutschen Schlagerwelt und mit ihr all die Zuhörer zu bestehen haben, benötigen sicher eine harte Gangart, selbst wenn diese Abenteuer darin bestehen, den Amazonas in Google Earth hinaufzuscrollen. Das ist natürlich nur ein schlechter Vergleich, die Bergsche Wirklichkeit sieht weitaus gefahrloser aus: Für ihr neues (Doppel-)Album, das vor kurzem erst den Hügel zum Platz Eins erklomm, ist sie ins Wasser gegangen (zwei schöne Antonyme übrigens: Berg Wasser), nachdem sie sich »zwei Jahre Zeit « genommen und »in unendliche Tiefen, unendliche Weiten hinausgewagt « hat - gemeinsam mit Dieter Bohlen, der sich »monatelang « am »Voll-Connecten zwischen Künstler und Publikum « abarbeitete. ${ }^{29}$ Dass Frau Berg sich für das Titelfoto von Atlantis dem Wagnis, ja Abenteuer ausgesetzt hat, unter Wasser die Luft anzuhalten, heißt nun nicht, dass die himmlischen Wolken und bewölkten Himmel ausgespielt haben. Es wird weiterhin geflogen und geschwebt in ihrer »magischen Traumwelt«, in der neben dem Lügen das Träumen das zweite große Trauma der Berg ist: Kaum ein Lied ohne Traum - alles Träumereien, versteht sich: Träume, die zerbrechen, Träume, die sich - das einzige Unsaubere weit und breit - auf »spür'n« und »verlier'n« reimen (»Träumer wie wir«). Für diese »verrückten Ideen« - so nennt Frau Berg ihre Texte - ist sie selbst zuständig, für das klangliche Surrounding ihr Produzent, zu dem man stehen mag, wie man will -: aber Dieter Bohlen weiß, wie musikalisches Stroh zu Platin gesponnen wird.

Bei Frau Berg dreht sich alles um das Problem-Duo Lieben/Lügen; bei den Amigos ist es das weitgehend problemfreie Pärchen Lieben / Leben, dem das Brüderpaar aus dem Hungener Stadtteil Villingen im Landkreis Gießen Jahr für Jahr eine erfolgreiche CD nach der anderen widmet - CDs, deren Cover sich allein in der Farbgebung unterscheiden und deren jeweils rund 15 Songs nicht nur noch langweiliger und vorhersehbarer als Fischer-Hinter-

»Top 10« des Jahres 2012 im Bereich »U-Musik« - noch vor >Dauerbrennern wie »Summertime«, »Take Me Home, Country Roads«, »As Time Goes By« usf. (cf. virtuos. Das Magazin der GEMA, H. 4, 2013, S. 36).

29 Alle Zitate stammen aus dem Promo-Video »Andrea Berg und Dieter Bohlen zurück im Studio« zur Veröffentlichung der Doppel-CD Atlantis; cf. http://www. youtube.com/watch?v=8-RIVrvPUMU (letzter Zugriff 5.4.2014). 
seer-Berg-Reim-Songs, sondern - dies das wahrhaft und zweifelsfrei Typische der Amigos-Songs - nahezu identisch sind. ${ }^{30}$ Ich habe vor kurzem eine detaillierte Fallstudie zu diesem Phänomen durchgeführt (Phleps 2014) und will mich hier nicht wiederholen, nur kurz anmerken, dass aus diesem Grunde Tanzmuckern die Amigos-Songs ein Gräuel sind: Sie können sie nicht unterscheiden und geraten dadurch in Schwierigkeiten.

Das Amigos-Album des Jahres 2013 thematisiert offen und offensiv die bereits angesprochene Vergreisung der Charts. Die »einzig wirklich langfristig erfolgreiche Boygroup « hält »der unwürdigen Diskussion um Überalterung und Jugendwahn den Spiegel vor «, erfahre ich aus dem Musikmarkt (az 2013), und in der Tat gehen die Dominatoren des Paralleluniversums »Deutsches Musikfernsehen« gleich im Titelsong der CD zur Sache:

Die Amigos: »Im Herzen jung «, 1. Strophe und Refrain

Viel erlebt, die Zeit ging schnell vorbei.

Wir waren jung und fühlten uns so frei.

Die Uhr des Lebens, die hält uns in Atem.

Es wird schon weitergeh'n, sind die Jahre auch vergangen.

In unserm Herzen sind wir für immer jung.

Und das Leben hat uns stark gemacht.

In unserm Herzen lebt die Erinnerung.

Glaub an die Liebe, sie hat schon Wunder vollbracht!

Wie so oft, geht den beiden gleichen Brüdern gegen Ende der Strophe der Reim aus, hier wohl infolge der »Uhr des Lebens« - wer hält da nicht den Atem an und fragt, wie das Lied weitergeht. Natürlich nicht wie Karl Valentins »Uhr von Loewe«, die gar nicht erst zum Vortrag gelangt -: Nein, wie so oft beginnt das Lieben/Leben-Duo durch den Refrain zu tanzen, bis das »Wunder«, ein weiterer Amigos-Refrain nämlich, »vollbracht « ist.

Aber es gibt in oder besser unter dieser unlogisch-logistischen Einfalt einen von Pop-Geschichte gesättigten Hintergrund, der dieses Wunderwerk amigoesker Reim- und Vortragskunst ganz neu zu beleuchten vermag. »Für immer jung « - bei den beiden befreundeten Brüdern als Operation am offenen Herzen durchgeführt - hat zwei musikalisch-inhaltliche Traditionsstränge, die direkt in unsere Inspektion zurückführen. 1984 quengelten die Münsteraner Synthie-Popper von Alphaville: »| want to be forever young «, was im Jahr 2000 der Tscheche Karel Gott »ein Leben lang für immer jung « ins Deutsche übertrug, was wiederum 2008 Bushido durch jugendgefährden-

30 Und durchweg in Dur stehen - ich habe im Amigos-Repertoire noch keine MollNummer entdecken können, auch wenn Bruder Bernd Ulrich behauptet, dann kenne man ihre Musik nicht (cf. Summen 2010). 
de Zeilen wie »Und dir bleibt nur deine Erinnerung, alles ist vergänglich« anreicherte. Und bereits 1974 ließ Bob Dylan auf seiner LP Planet Waves zwei Versionen eines anderen, nämlich seines »Forever Young « aufeinanderfolgen, was wiederum im Bushido-Jahr 2008 der inzwischen mehrfache Bundesverdienstkreuzträger Wolfgang Niedecken mit seinen BAP ins FantasyKölsch transferierte, nicht ohne Dylans »May your heart always be joyful / And may your song always be sung / May you stay forever young « ins TypischDeutsche zu wenden: »Auf dass dein Herz immer in Dur [Sie erinnern sich: in Dur!] schlägt, / seinen Takt hält, seinen Schwung, / dann bleibst du für immer jung. « ${ }^{31}$ So einfach ist das - und so wird man Zosamme alt, wie das neuste Album des deutschen Zimmerman sich nennt - es gelangte Ende September 2013 immerhin auf Patz 4 der deutschen Album-Charts.

Auf Platz Eins stand fünf Wochen zuvor - und zwar an der Spitze einer außergewöhnlichen und nur durch die populär-klassikalen Highlights eines unbedeutenden und längst verstorbenen italienischen Komponisten gestörten, rein deutschen Konstellation:

Platz 1-10 der Album-Charts 2013, 34. Woche (23.-29. August 2013) ${ }^{32}$

1 Helge Schneider: Sommer, Sonne, Kaktus!

2 Santiano: Mit den Gezeiten

3 Mono Inc.: Nimmermehr

4 Xavier Naidoo: Bei meiner Seele

5 Alligatoah: Triebwerke

6 Anna Netrebko, Gianandrea Noseda \& Orchestra Del Teatro Regio Di Torino: Verdi

7 Santiano: Bis ans Ende der Welt

8 Helene Fischer: Best of

9 Cro: Raop

10 Sportfreunde Stiller: New York, Rio, Rosenheim

Auf Platz Eins stand in dieser 34. Woche die einzige, die große, die großartige Überraschung der deutschen Album-Charts 2013: Helge Schneiders Sommer, Sonne, Kaktus - Ausrufezeichen! Ein Album, das in nur drei deutsch gesungenen Liedern (die übrigen elf sind auf Englisch vorgetragen, nicht wenige davon in typisch Schneiderscher 1960er-Jahre-Zahnklammer-

31 0-Ton Niedecken: »Op dat dieh Häzz immer enn Dur schläät, / singe Takt hällt, singe Schwung, / dann blievs du für immer jung. « Dialekt-Transkription wie die Umschrift ins Hochdeutsche finden sich auf der offiziellen BAP-Homepage; cf. http://www.bap.de/start/musik/songtexte/titel/für-immer-jung-foreveryoung (letzter Zugriff 5.4.2014).

32 Cf.http://de.wikipedia.org/wiki/Liste_der_Nummer-eins-Alben_in_Deutschland (letzter Zugriff 5.4.2014). 
Aussprache, andere - wie »Drinking Blues« - mit fast schon Waitsschen Dimensionen) die Themen und Musiken der Charts zu sich selbst kommen lässt, d.h. in ein musikalisch-inhaltliches Eldorado führt, wo allerdings keine Gold- und Platinströme fließen, sondern Tränen eines herzhaften, in keinem Halse stecken bleibenden Lachens - so viel Pathos muss sein!

Im ersten, dem auf Sommerhit gepolten Titelsong werden noch - wie in den Album-Charts - ein paar Brocken, ja eher Überreste der internationalen Popsprache eingestreut, die Aussprache freilich ist schon mangelhaft. ${ }^{33}$ In Nummer 2 (»Nachtigall, huh«) sind die Verständigungs- oder besser Verständnisschwierigkeiten mit der Popwelt gravierender: „Opa Gander-Style « ruft Helge Schneider, noch und nochmal »Opa Gander-Style« und meint natürlich in dieser gleichsam ebenerdigen Psy-Adaption nichts anderes als: »Oppa ${ }^{34}$ Gangnam Style«.

»Offenes Hemd«, der dritte deutschsprachige Song, betreibt offensichtlich die Rehabilitation eines der größten musikalischen Marterinstrumente für Generationen deutscher Schüler -: der Blockflöte. Im dazugehörigen Video gibt Schneider den Jürgen Drews oder einen anderen der inzwischen ins Greisenalter gekommenen Schlager-Schwiegersöhne, deren offene, d.h. aufgeknöpfte Hemden das Äußerste und zugleich äußerst Peinliche an Freizügigkeit markierten. Inhaltlich wird die gesamte Palette des Schlagerliebens durchgespielt: Verlieben-Lieben-Entlieben-Wiederlieben, ohne dass das Schlagerwort Nummer Eins auch nur fällt oder dem Zuhörer beim Hören auch nur einfällt. Die erste Strophe bietet eine Engführung, alle folgenden deklinieren die Stationen durch, die schönste davon nobilitiert - was fast unmöglich scheint - den Frauennamen Jutta.

33 Während die Shindys und Bushidos dieser Welt ihre Beschäftigungslosigkeit durch ständiges Dampfablassen resp. grundloses Stressmachen überkompensieren müssen, hat in »Sommer, Sonne, Kaktus« der (mit Arbeit verbundene) Alltag ein paar Tage frei bekommen: »Never, never go to work, lieber holiday« was natürlich nicht Nichtstun bedeutet, sondern am Strand spielen (bspw. »Federbäll«), den "girls on the $\mathrm{P}_{0}$ « schauen und Paella essen, wie es »the Brauch « ist - ein Wunschkonzert des kleinen Mannes, der im dazugehörigen Video Treppen herabschreitet und sich von Frauen umkreisen lässt - wie es in jeder Schlagershow (fast hätte ich zweitklassigen geschrieben - aber erstklassige gibt es ja nicht) >the Brauch ist. Aber wir sind weder im deutschen Schlager, noch in einem seiner Musentempel gepflegter Langeweile und alles ist eine Schneidersche »llusion! «, die im Duisburger Hallenbad zerbricht.

34 Möglicherweise ruft Schneider sogar - statt dieser lautmalerischen Transkription des Koreanischen - »Obacht: Gangnam Style! . 
Schneider: »Offenes Hemd «, 1. und 2. Strophe

Offenes Hemd, und der Wind streift meine Haut

Gestern noch einsam, heute schon vertraut

Wie kann das geh'n? Auf Wiederseh'n!

Ah-jäh-jeh oh-o-o-oh oh-o-oh.

Offenes Hemd, und der Wind weht durch dein Haar, na-na-na.

Ich weiß noch, wie es war,

als ich dich angesprochen hab'.

Ich sagte: »Wie heißt du denn?«

Sie sagte: »Jutta!« — Da war alles klar.

Und während die gesamte Pop- und Schlagerwelt autogetuned wird, spielt Schneider vokal wie instrumental den Hyperdilettanten - und textlich den Arglos-Unerfahrenen, dessen Vorstellung von Sex gerade mal bis zum »Knutschen« reicht und dessen Unanständigstes aus kindlichen Fäkalausdrücken besteht, die sich zudem noch eher zufällig aus einer Art Scat-Rapping ergeben:

Helge Schneider: »Sommer, Sonne, Kaktus«, Schluss

Sommer, Sonne, Kaktus -

Kaktus, Kaktus, Kak-kak-kak-kaktus,

Ka-ka-ka-kaktus, Kaktus, Ka-ka-ka-kaktus,

Ka-kaktus, Ka-ka-ka-ka-ka-kaktus,

Kaktus, Kaktus, Ka-ka-ka-kaktus,

Kaktus, Kaktus, Ka-ka-ka-ka-kaktus usf.

Natürlich gäbe es noch vieles über Schneiders Erfolgsalbum zu berichten und natürlich ist auch hier nicht alles eitel Sommer/Sonne, sondern manches auch ganz schön Kaktus, beispielsweise Schneiders arg mit Patina überzogene Jazz-Exkursionen, die man inm aber - auch wenn er sie ernster nimmt als sich selbst - verzeiht, weil es ihm gelungen ist, überhaupt Musik jenseits des Mainstream, andere Musik als die knäckebrotartige in die Charts - man könnte fast sagen: zu schmuggeln.

Diese Schmuggelei ${ }^{35}$ ist nicht zu verwechseln mit dem Schmuh um das »verbotene Album« resp. der musikindustriellen Revitalisierung der altvor-

35 Um die Schmuggelei sogleich wieder zu relativieren: Auf Sidos Nummer-EinsAlbum der 50. Woche des Jahres 2013, 30-11-80, >begegnen< sich Helge Schneider und Paul Hartmut Würdig alias Sido »Hammer fett, Bombe krass« im DuoRap »Arbeit « mit den ohrwurmlastigen Mehrfachzeilen »Was ist nur los in diesem Land? / Alle gehen arbeiten, nur ich nicht « im Hook und der Schneiderschen Conclusio »Arbeit ist gut - wenn sie ganz weit weg ist«. Der doppelte Boden ist textlich natürlich durchgängig offensichtlich, musikalisch freilich... 
deren Marke Heino als »freundlich grüßenden« Altrocker. Trotz eindeutig karnevalesker Züge bleibt hier das Lachen unweigerlich im sprichwörtlichen Halse stecken, zumal es - ebenfalls wie beim deutschen Karneval - auch gar nicht mitgedacht ist. Am stramm humorfreien Heino wie den anderen >Gipfelstürmern< des Jahres 2013 zeigt sich auch, dass typisch deutsche Charaktermasken oder besser: Charaktermasken des Typisch-Deutschen nicht allein uneinheitlich und vielfältig (freilich nicht vielschichtig, -seitig oder gar -deutig, sondern im so klanglosen wie treffenden Sinne vielfarbig) sind, sondern auch verschoben, neu justiert werden können. Sie werden ebenso neu- wie re-konstruiert, d.h. das Typisch-Deutsche scheint sich ständig neu zu erfinden - ohne dass sich freilich etwas Neues ereignet oder in den >Erfindungen< irgendetwas Neues findet. Es ist die - von Schneider in seinem Sommer/Sonne-Hit herbeigerufene - »llusion « von Veränderung, die die Iteration des Immergleichen verdeckt, es ist die Fassade, hinter der sich nichts verbirgt, es ist das Deutsche, das typisch zu sein vorgibt.

Und nicht zuletzt ist »zwischen >deutsch < und >typisch deutsch««, wie schon Hermann Bausinger (2000: 17) zu Recht anmerkte, »der Unterschied nicht allzu groß«, ja eigentlich nicht vorhanden, da »jeder Benennung eine Typisierung zugrunde « liegt. Und so kann ich Ihnen versichern, dass jegliche Chartliste aus Deutschland typisch deutsch ist - auch die, in denen keine deutschsprachigen Titel und keine deutschen Musiker auftauchen, was realiter nicht gegeben ist, ${ }^{36}$ aber angesichts meiner hoffnungslosen Befunde und Befindlichkeit nicht eben die schlechteste Option zu sein scheint.

36 Und selbst unter den ersten 22 Musikern mit den meisten Nummer Eins-Alben in Deutschland (http://de.wikipedia.org/wiki/Liste_der_Nummer-eins-Alben_in_ Deutschland, letzter Zugriff 5.4.2014) finden sich neben den weltweit erfolgreichen Beatles, Stones, Abba, Madonna, Michael Jackson, Metallica... mehr als die Hälfte, nämlich 12 typisch deutsche Vertreter, darunter die bereits erwähnten Andrea Berg, BAP, Grönemeyer, aber auch Xavier Naidoo, Rammstein, Die Toten Hosen und ganz oben auf Platz 2 James Last und auf Platz 1 Peter Maffay - was nicht zuletzt deutlich macht, dass ich es bei meiner zufälligen Listenwahl noch schlimmer hätte treffen können. 


\section{Literatur}

az (2013). »Einzig langfristig erfolgreiche Boygroup.«In: Musikmarkt, H. 22, S. 65.

Bausinger, Hermann (2000). Typisch deutsch. Wie deutsch sind die Deutschen? München: C.H. Beck.

BPjM [Bundesprüfstelle für jugendgefährdende Medien] (2013). »Rap-CD $>N W A<$ des Interpreten s Shindy< indiziert. Vorläufige Listenaufnahme. OPr. 629/13; BPjMEntscheidung Nr. VA1/13 vom 16.07.2013. «In: BPJM-Aktuell, H. 3, S. 3-20.

Brecht, Bertolt (1967). »Das Typische. In: Ders., Gesammelte Werke 19. Schriften zur Literatur und Kunst 2. Hg. v. Suhrkamp Verlag in Zusammenarbeit mit Elisabeth Hauptmann. Frankfurt/M.: Suhrkamp, S. 531.

Büchmann, Georg (1941). Geflügelte Worte. Der Zitatenschatz des deutschen Volkes. Berlin: Haude \& Spenersche Verlagsbuchhandlung Max Paschke (Volksausgabe).

Costazza, Alessandro (2006). »Das >Charakteristische< ist das >Idealische <. Über die Quellen einer umstrittenen Kategorie der italienischen und deutschen Ästhetik zwischen Aufklärung, Klassik und Romantik. « In: Goethezeitportal; http:// www.goethezeitportal.de/db/wiss/epoche/costazza_charakteristische.pdf (letzter Zugriff 5.4.2014).

fro (2013). »Bushido: Diese Partei wähle ich am Sonntag. «In: merkur-online.de vom 16. September; http://www.merkur-online.de/aktuelles/politik/bundestagswah l-2013/bushido-diese-partei-waehle-sonntag-zr-3114402.html (letzter Zugriff 5. 4.2014).

Fromm, Dani (2013). »R.I.P. - Rest In Pussy« [laut.de-Kritik zur CD Lil Wayne: I Am Not A Human Being II]. In: laut.de; http://www.laut.de/Lil-Wayne/Alben/I-AmNot-A-Human-Being-II-89080 (letzter Zugriff 5.4.2014).

Gerhard, Anselm (2006). „Die >Vorherrschaft der deutschen Musik nach 1945 eine Ironie der Geschichte. «In: Deutsche Leitkultur Musik? Zur Musikgeschichte nach dem Holocaust. Hg. v. Albrecht Riethmüller. Stuttgart: Franz Steiner, S. 13-28.

Gothe-Syren, Oliver (2010). "Schlagersänger Matthias Reim nach 4 Jahren aus der Insolvenz raus. «In: Insolvenz-News vom 20. April; http://www.insolvenz-news. de/schlagersaenger-matthias-reim-nach-4-jahren-aus-der-insolvenz-raus (letzter Zugriff 5.4.2014).

Hirt, Aloysius (2008). "Laokoon." In: Ästhetik des Charakteristischen. Quellentexte zu Kunstkritik und Streitkultur in Klassizismus und Romantik. Hg. v. Roland Kanz und Jürgen Schönwälder (= Super alta perennis. Studien zur Wirkung der Klassischen Antike, Bd. 3). Bonn: University Press bei V\&R unipress, S. 33-46 (orig. in Die Horen. Hg. v. Friedrich Schiller. 3. Jg., 1797, 10. Stück, S. 1-26).

Jüttner, Julia (2007). »Matthias Reim: Verdammt, ich mach mich lächerlich!« In: Spiegel Online vom 23. August; http://www.spiegel.de/panorama/leute/matt hias-reim-verdammt-ich-mach-mich-laecherlich-a-501427.html (letzter Zugriff 5.4.2014).

Korn, Julia (2011). »Deutsch-Tunesier Bushido bekommt den BAMBI für Integration. « In: Hubert Burda Media vom 7. November; http://www.hubert-burda-me dia.de/newsroom/mitteilungen/-deutsch-tunesier-bushido-bekommt-den-bambifuer-integration_aid_71582.html (letzter Zugriff 5.4.2014).

Kuboth, Sebastian (2005). »Frei.Wild« [Interview mit Philipp Burger]. In: punkrocknews.de vom 1. August; http://www.punkrocknews.de/interviews/9/frei-wild (letzter Zugriff 17.12.2013). 
Lukács, Georg (1967). Über die Besonderheit als Kategorie der Ästhetik. Darmstadt: Luchterhand.

Marx, Karl (1973). Das Kapital. Kritik der politischen Ökonomie. Erster Band (= Karl Marx / Friedrich Engels Werke, Bd. 23). Berlin: Dietz.

Nietzsche, Friedrich (1967a). "Götzen-Dämmerung oder Wie man mit dem Hammer philosophiert." In: Ders., Werke in zwei Bänden. Bd. II. Hg. v. Ivo Frenzel. München: Hanser, S. 323-397 [orig. Leipzig: C.G. Naumann 1889].

Nietzsche, Friedrich (1967b). »Jenseits von Gut und Böse. Vorspiel einer Philosophie der Zukunft.«In: Ders., Werke in zwei Bänden. Bd. II. Hg. v. Ivo Frenzel. München: Hanser, S. 9-173 [orig. Leipzig: C.G. Naumann 1886].

Phleps, Thomas (2014). »Freunde bis ans Ende der Zeit - Schlagertümlichkeit reloaded.«In: Rollenspiele. Musikpädagogik zwischen Bühne, Popkultur und Wissenschaft. Festschrift für Mechthild von Schoenebeck zum 65. Geburtstag. Hg. v. Thomas Erlach und Burkhard Sauerwald. Frankfurt/M.: Peter Lang, S. 433-450.

Sponheuer, Bernd (2001). „Über das >Deutsche< in der Musik. Versuch einer idealtypischen Rekonstruktion. « In: Deutsche Meister - böse Geister? Nationale Selbstfindung in der Musik. Hg. v. Hermann Danuser und Herfried Münkler. Schliengen: Edition Argus, S. 123-150.

stern exklusiv (2013). »Bushido und die Mafia. Insider Kay One packt aus.« In: stern.de vom 9. Oktober; http://www.stern.de/panorama/stern-exklusiv-bushi do-und-die-mafia-insider-kay-one-packt-aus-2063446.html (letzter Zugriff 5.4. 2014).

Summen, Maurice (2010). »>Eine Sauerei, unsere Musik niederzumachen<. Sänger Bernd Ulrich von den >Amigos‘, Deutschlands erfolgreichstem Schlagerduo, über Karriere per Teleshopping, Lieder über Kindesmissbrauch und die Häme vieler Musikkritiker«. In: Frankfurter Rundschau vom 12. Januar, S. 20f.; http://www. fr-online.de/panorama/amigos-saenger-im-interview--sauerei--unsere-musik-nie derzumachen-,1472782,3162146.html (letzter Zugriff 5.4.2014).

Summen, Maurice (2013). » Freiwild? Kenn ich nicht! < Heino über sein neues Album und den überraschenden Erfolg bei der jungen Generation.« In: Frankfurter Rundschau vom 29. April, S. 32.

Wagner, Richard (1914). »Deutsche Kunst und deutsche Politik« [1867/68]. In: Ders., Gesammelte Schriften und Dichtungen. Bd. 8. Hg. v. Wolfgang Golther. Leipzig: Deutsches Verlagshaus Bong \& Co, S. 30-124.

Weber, Max (1991). „Die >Objektivität sozialwissenschaftlicher und sozialpolitischer Erkenntnis. « In: Ders., Schriften zur Wissenschaftslehre. Stuttgart: Reclam, S. 21-101 (orig. in: Archiv für Sozialwissenschaft und Sozialpolitik 19, 1904 , S. 22-87).

Weimer, Sophia / Vogel, Elke (2013). »Gala - lalala. Beinahe fehlerfrei ging die 65. Bambi-Verleihung über die Bühne. «In: Frankfurter Rundschau vom 16./17. November, S. 46. 


\title{
Diskographie
}

Alphaville (1984). »Forever Young. « Single, WEA 249 264-7.

BAP (2008). »Für immer jung. "Auf: Radio Pandora - Plugged. CD, Capitol 50999 20903824.

Berg, Andrea (2013). »Du hast mich tausendmal belogen. « Auf: Abenteuer - 20 Jahre Andrea Berg. Box-Set, Sony Music 88765433242.

Berg, Andrea (2013). »Träumer wie wir«. Auf: Atlantis. 2-CD, Sony Music/Ariola 88883764862.

Bushido feat. Karel Gott (2008). „Für immer jung.« Single, ersguterjunge 088697436972.

Die Amigos (2013). »Im Herzen jung. « Auf: Im Herzen jung. CD, VM Records CD 171.148.

Dylan, Bob (1974). »Forever Young.« Auf: Planet Waves. LP, Asylum Records AS 53003.

Genetikk (2013). »D.N.A. « Auf: D.N.A. CD, Selfmade Records SELF034.

Gott, Karel (2000). »Für immer jung.« Auf: Für immer jung. CD, Polydor 543 525-2.

Grönemeyer, Herbert (1984). »Männer. «Single, EMI 1C 0061469067.

Heino (2013). »Junge. « Auf: Mit freundlichen Grüßen. CD, Sony 88725460672.

Lindenberg, Udo (1981). »No Future?« Auf: Rock ' $n$ ' Roll und Rebellion. Ein panisches Panorama. EP, Telefunken 66.20217.

Prinz Pi (2013). „Kompass ohne Norden. " Auf: Kompass ohne Norden. CD, Keine Liebe Records KLR007.

RAF 3.0 (2013). »Zum Quadrat.« Auf: Hoch 2. CD, Indipendenza Indi002.

Reim, Matthias (2013). »Wir sind unendlich.« Auf: Unendlich. CD, Electrola 5099943381722.

Schneider, Helge (2013). Sommer, Sonne, Kaktus!. CD, Polydor 0602537447275.

Shindy feat. Bushido (2013). »Stress ohne Grund. « Auf: NWA. CD, AMF Management 08888-3741422.

Sido ft. Helge Schneider (2013). »Arbeit.« Auf: 30-11-80. CD, Vertigo 3753149.

\begin{abstract}
Subsequent to a critical approach to the concept of the »typical« this paper seeks to work out the diversity as well as the simplicity, the manifoldness as well as the simple-mindedness of current German popular music on the basis of the German album charts of 2013. The present study focuses on varieties of - obviously youthorientated - German hip-hop (Shindy, Bushido...), on timeless phenomena of German Schlager music (Andrea Berg, Amigos...) and not least on artists who cross the borders in terms of content and music (Helge Schneider).
\end{abstract}

